
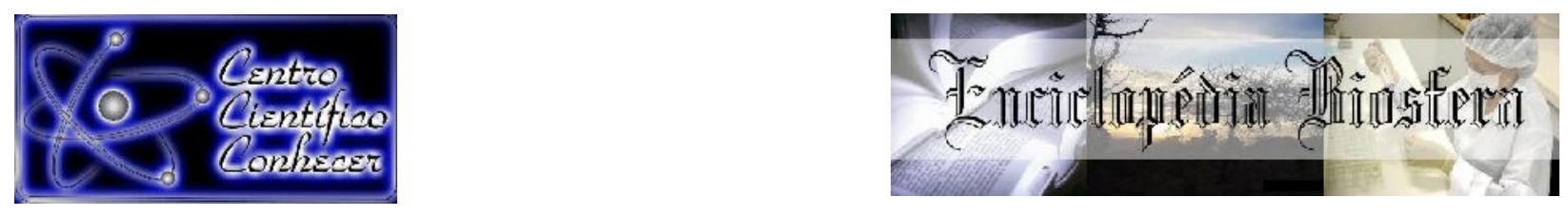

\title{
\#PROMOÇÃOdASAÚDE: ANÁLISE DAS PUBLICAÇÕES RELACIONADAS À PROMOÇÃO DA SAÚDE NO INSTAGRAM
}

Elen Bruna Pereira Vicente ${ }^{1}$;Filipe Zemuner Paiva Rossini 2; Tânia Maria Gomes da Silva $^{3}$, Marcelo Picinin Bernuci ${ }^{4}$

${ }^{1}$ Mestra pelo programa de Pós-graduação em Promoção da Saúde. Bolsista PROSUP/CAPES. Universidade de Maringá-UniCesumar. Maringá, Paraná, Brasil

${ }^{2}$ Graduando em Medicina. Universidade de Maringá- UniCesumar -Maringá, Paraná, Brasil.

${ }^{3}$ Professora Doutora do Programa de Pós-Graduação em Promoção da Saúde da Universidade de Maringá (UniCesumar) - Maringá-PR. Pesquisadora do Instituto

Cesumar de Ciência, Tecnologia e Inovação, ICETI, Maringá, Paraná tania.gomes@unicesumar.edu.br

${ }^{4}$ Professor Doutor do Programa de Pós-Graduação em Promoção da Saúde, UniCesumar, Maringá-PR, Pesquisador do Instituto Cesumar de Ciência, Tecnologia e Inovação, ICETI, Maringá, Paraná.

mpbernuci@gmail.com

Recebido em: 15/02/2021 - Aprovado em: 15/03/2021 - Publicado em: 30/03/2021 DOI: 10.18677/EnciBio_2021A18

\begin{abstract}
RESUMO
A Rede Social Online Instagram se destaca como a principal rede de compartilhamento de imagens e vídeos, permitindo expressiva interatividade social, com aplicações em diferentes campos. Estudos recentes têm mostrado que esse ambiente digital pode ser utilizado como importante instrumento para a propagação de estratégias de promoção da saúde. O presente estudo, de caráter descritivo, objetivou classificar as postagens mais relevantes, segundo plug-in do Instagram, indexada pela hashtag promoção da saúde. Realizado em setembro de 2019, o estudo evidenciou que das 100 postagens analisadas, 67\% correspondiam a imagens com texto; $26 \%$ a fotos; $5 \%$ a vídeos e $2 \%$ a imagens sem textos. A maioria das postagens eram relacionadas a prevenção contra doenças $(46 \%)$, alimentação/nutrição (41\%) e atividade física (13\%). Percebeu-se, ainda, que a maioria das publicações com o uso de "\#promoçãodasaúde" foram realizadas realizada pelo Ministério da Saúde (63\%), por profissionais da saúde (35\%) e, minoritariamente, por pessoas não ligadas à área da saúde (2\%). A partir da perspectiva da educação em saúde por meios digitais, defende-se a potencialidade do Instagram para transmitir informações, gerar mudanças e promover saúde, notadamente quando utilizada por profissionais e órgãos do setor.
\end{abstract}

PALAVRAS-CHAVE: Educação em saúde; Promoção da saúde; Redes sociais online. 


\title{
\# HEALTH PROMOTION: ANALYSIS OF INSTAGRAM HEALTH PROMOTION PUBLICATIONS
}

\begin{abstract}
The Online Social Network Instagram stands out as the leading network for image and video sharing with applications in the field of health promotion. Recent studies have shown that this social network can be used as an important tool for promoting healthy habits with potential to be used in health promotion strategies. Thus, we aim to classify Instagram posts related to health promotion. This is a descriptive study conducted in September 2019 based on the analysis of the 100 most relevant Portuguese-language posts, according to the Instagram plug-in, indexed by the health promotion hashtag. It was found that of the 100 posts analyzed $67 \%$ correspond to images with text; $26 \%$ to photos; $5 \%$ to videos and $2 \%$ to image. Most posts are related to disease prevention (46\%), followed by food / nutrition (41\%) and physical activity (13\%). In addition, most publications using "\#health promotion" were carried out by the Ministry of Health (63\%) and health professionals (35\%), the minority $(2 \%)$ by ordinary people. Instagram has been serving content about health promotion and is often used by health professionals and agencies.
\end{abstract}

KEYWORDS: Health education; Health promotion; Online social networks.

\section{INTRODUÇÃO}

A promoção da saúde baseia-se em estratégias capazes de suprir as necessidades sociais de saúde, tanto no aspecto individual como no coletivo (MALTA et al., 2018). Não deve ser pensada como apenas uma questão de educação, mas entendida como um conhecimento que só se valida quando efetivamente vertido em prática, levando em consideração a realidade específica de cada país, estado ou nação. Portanto, não é responsabilidade apenas do setor de saúde, uma vez que outros componentes sociais têm impacto significativo nessas ações, como as condições que as pessoas nascem, crescem, vivem, trabalham e envelhecem, as experiências individuais, o meio ambiente e o acesso a serviços de prevenção e tratamento de doenças (ABREU et al., 2018).

A Constituição Federal de 1988 institucionalizou a promoção da saúde no Brasil (YAMAGUCHI et al., 2016), mas, passados todos esses anos, o cenário aparente é desolador, considerando-se as desigualdades de poder, riqueza, gênero, raça/etnia, educação, urbanização, entre outros determinantes sociais que evidenciam as desigualdades que vigoram no País (LA PEÑA, 2019). Além disso, o pouco investimento no Sistema Único de Saúde, que tem levado a um sistemático sucateamento da rede pública, deve ser pensado como uma ação que inibe o desenvolvimento de práticas promotoras da saúde (ISCHKANIAN; PELICIONI, 2019).

Dentre os princípios e objetivos específicos adotados pela Política Nacional de Promoção da Saúde (PNPS) está o empoderamento, que discorre sobre o processo de estimular coletivamente as pessoas a obterem a autonomia e controle de suas escolhas para tomada de decisões através do desenvolvimento de habilidades voltadas para promoção da saúde (BRASIL, 2014). Vindo ao encontro das estratégias de empoderamento em saúde, destaca-se a literacia em saúde, definida como a capacidade de se obter e compreender informações básicas 
relacionadas à saúde para a tomada de decisões conscientes (QUEMELO et al., 2017). Assim, para haver a promoção da saúde de forma efetiva, os sujeitos necessitam agir a partir de uma escolha pessoal e não de uma imposição (TENGLAND, 2007; CHIAPPERINO; TENGLAND, 2015), o que faz da comunicação em saúde um campo diacronicamente articulado aos das políticas públicas, educação popular, movimentos sociais, direitos humanos, etc.

Dentre as formas de comunicação em saúde adotadas na contemporaneidade, se destacam as Redes sociais Online (RSO), como uma nova ferramenta de interação entre as pessoas, permitindo a comunicação de forma rápida para todas as partes mundo (MOREIRA; PINHEIRO, 2015; MARCELINO et al., 2016). Entre as RSO, o Instagram ganhou rápida popularidade por permitir aos usuários a captura e o compartilhamento instantâneo de seus momentos de vida com amigos e familiares, através de imagens e vídeos, complementadas pelo uso de emojis, pictogramas que substituem as mensagens de texto. O Instagram é bastante popular no Brasil. Em 2016, 75\% dos brasileiros faziam uso desse instrumento virtual, número superior à média global de 32\%. Além disso, no País, essa rede social não está circunscrita ao público jovem, mas atrai também indivíduos na faixa dos 55 aos 65 anos, que representam 57\% dos usuários (APROBATO, 2018).

Consciente de que, entre as RSO, o Instagram ocupa a segunda posição na preferência dos brasileiros, o Ministério da Saúde (MS) criou, em 2010, a sua página, buscando a interação com os usuários por meio do compartilhamento na publicação de imagens e vídeos (AGUIAR, 2018). O objetivo da página do MS no Instagram é a qualificação do Sistema Único de Saúde, por meio do diálogo com a população. Em 2017, a página contava com 622 mil seguidores (PARACKAL et al., 2017; BILOTTI et al., 2017).

Diante da importância de políticas públicas bem definidas de promoção da saúde e do entendimento de que comunicação e empoderamento são princípios estratégicos para estimular ações conscientes de autocuidado em saúde, objetivouse no presente artigo apresentar uma análise das postagens de conteúdos de promoção da saúde no Instagram, a fim de analisar a viabilidade dessa RSO como um veículo de informação de saúde para a população.

\section{Delineamento experimental e coleta dos dados}

\section{MATERIAL E MÉTODOS}

Tratou-se de um estudo descritivo realizado na RSO Instagram durante o mês de setembro de 2019 com foco na análise de publicações públicas de língua portuguesa mais relevantes indexadas na hashtag promoção da saúde. Embora os usuários possam fazer publicações públicas ou privadas no Instagram, apenas postagens públicas foram selecionadas para o estudo.

Postagens públicas aparecem em um fluxo de mensagens quando uma hashtag específica é usada. As hashtags permitem que os indivíduos marquem suas postagens com palavras específicas que outros usuários podem pesquisar. O fluxo delas destaca as mais recentes com a hashtag. No presente estudo, foi utilizada a \#promoçãodasaúde com a maior quantidade de publicações. O refinamento adicional da amostra foi feito com um recurso do Instagram que localiza os 100 posts mais relevantes em um fluxo de hashtags escolhido. 


\section{Análise dos dados}

A análise das postagens foi realizada manualmente por dois pesquisadores independentes a fim de organizar tematicamente o conteúdo bem como determinar a origem das mesmas $e$ as repercussões entre os instagramers. Os dados quantitativos foram analisados por meio de estatística descritiva e apresentados em tabelas de distribuição de frequências. Utilizou-se a frequência absoluta e a porcentagem para as variáveis categóricas. Todas as análises estatísticas foram realizadas com o auxílio do ambiente estatístico R ( $R$ Development Core Team), Versão 3.3.1.

\section{Caracterizacão das postagens}

\section{RESULTADOS E DISCUSSÃO}

O gráfico 1 apresenta os dados referentes à categorização das postagens segundo o tipo de mídia (imagem, imagem com texto, foto e vídeo) para cada hashtag utilizada. Notou-se que das 100 postagens analisadas, oriundas da "\#promoçãodasaúde" utilizada no estudo, 2\% correspondiam a imagens; $67 \%$ a imagens com texto; $26 \%$ a fotos; e $5 \%$ a vídeos.

GRÁFICO 1. Postagens segundo o tipo de mídia.

\section{Postagens segundo o tipo de mídia}

$\square$ Imagem $\square$ Imagem com texto $\square$ Foto $\square$ Vídeo

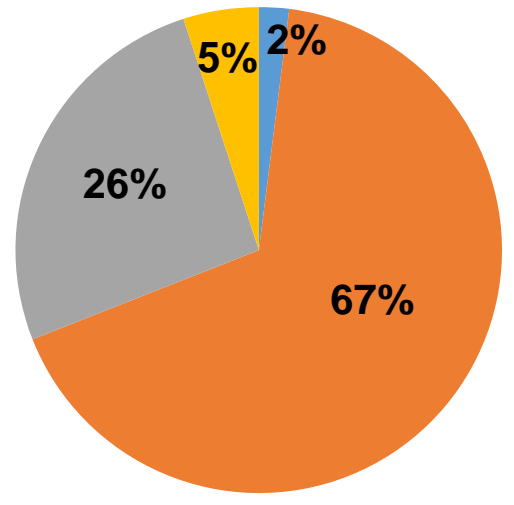

Fonte: pesquisa dos autores (2020)

O gráfico 2 apresenta os dados relacionados às postagens segundo o conteúdo. Os conteúdos foram organizados em três categorias: 1) alimentação/nutrição (postagens relacionadas à dieta, alimentos e receitas); 2) atividade física (postagens relacionadas a esportes e práticas de exercícios físicos); 3) prevenção de doenças (postagens relacionadas a estratégias para prevenção de doenças e promoção da saúde). Notou-se que das 100 postagens analisadas a maioria $(46 \%)$ relacionou-se a conteúdos de prevenção contra doenças; seguida por alimentação (38\%) e atividade física (13\%). 
GRÁFICO 2. Postagens segundo o conteúdo.

\section{Postagens segundo o contéudo}

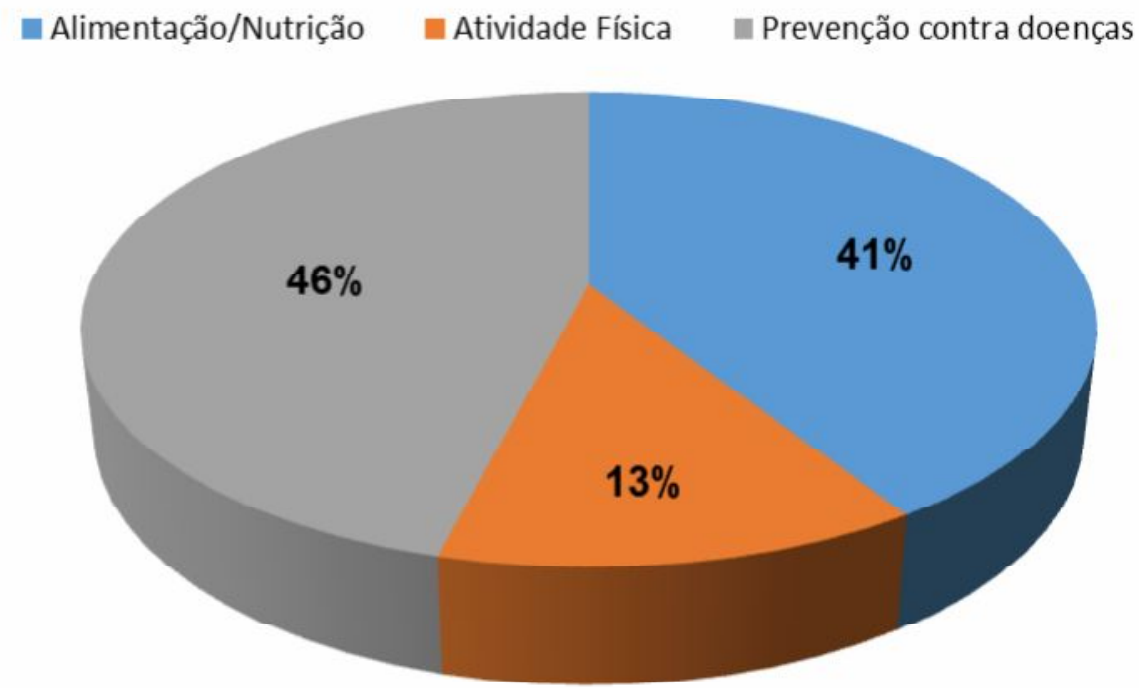

Fonte: pesquisa dos autores (2020)

\section{Origem das postagens}

Baseado nas informações públicas contidas nas contas do Instagram, as postagens foram organizadas em três categorias, segundo dados do instagramer que efetuou a postagem: profissionais de saúde, pessoas comuns (usuários do Instagram que não são profissionais de saúde) e Ministério da Saúde. Das 100 postagens analisadas, $63 \%$ foi postada pelo Ministério da Saúde; 35\% por profissionais da saúde e apenas $2 \%$ por pessoas comuns, o que sugere a veracidade das informações publicadas (gráfico 3).

GRÁFICO 03. Origem das postagens.

\section{Origem das Postagens}

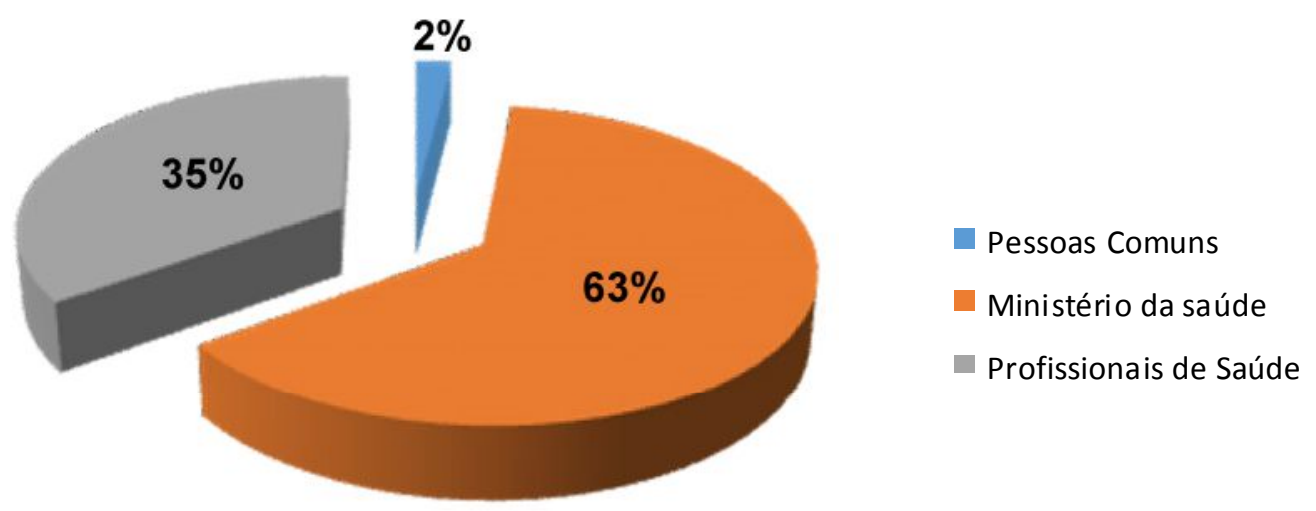

Fonte: pesquisa dos autores (2020)

ENCICLOPÉDIA BIOSFERA, Centro Científico Conhecer - Jandaia-GO, v.18 n.35; p. 246 
O poder da mídia em seus múltiplos formatos (jornais, revistas, rádio, TV) e sua influência sobre as massas tem sido conduzida, muitas vezes, numa perspectiva crítica, ressaltando-se seu caráter de manipulação partidária e ideológica, indiferente às demandas dos grupos de menor capital econômico, e seus efeitos de homogeneização cultural na sociedade capitalista (CASTELLS, 2000). Estudos mais recentes vêm confirmando esta proposição, validando-a, inclusive, no que diz respeito às mídias digitais, consideradas um meio dos mais eficientes para exercer estas influências (LIRA et al., 2017). Mas os meios de comunicação podem também ser utilizados para discursos contra-hegemônicos e, nesse aspecto vale ressaltar a contribuição das mídias digitais para a democratização do discurso, possibilitando ações de interesse comunitário (BARROS, 2018).

$\mathrm{Na}$ área da saúde, a internet tem possibilitado várias ações pedagógicas de promoção da saúde, notadamente por permitir intervenções simples e econômicas, eliminando as barreiras físicas e temporais (CARVALHO; GASTALDO, 2008; LIMA et al., 2015; MARCELINO et al., 2016; CARVALHO et al., 2017). Para Serradilha et al. (2019), essas atividades de promoção da saúde contribuem para melhorar a qualidade de vida das pessoas, reduzindo as fragilidades, incentivando à autonomia, ao mesmo tempo em que também influenciam a prática dos profissionais da saúde.

Considerando-se a potencialidade das mídias digitais para disseminar informações e estimular conhecimento, buscou-se mostrar neste estudo que postagens relacionadas ao tema promoção da saúde são frequentes no Instagram. Vale ressaltar que, como as RSO, a exemplo do Instagram, permitem que as informações sejam publicadas em tempo real, é preciso atenção ao conteúdo propagado, pois esses podem intervir tanto de maneira benéfica quanto maléfica na educação em saúde do usuário e sua utilização como ferramenta de intervenção de promoção de saúde deve ser analisada com cuidado (HOLMBERG et al., 2018), visando a efetiva educação e empoderamento dos usuários desse meio de comunicação digital.

\section{CONCLUSÃO}

Embora o estudo não tivesse como propósito avaliar a qualidade das postagens, concluiu-se que o fato de que a maioria fosse realizada pelo MS e por profissionais da saúde assegura, de certa forma, a qualidade das mesmas. Ao utilizaram o Instagram para propagar conteúdos relacionados a como promover saúde, o Ministério da Saúde e os profissionais ligados a este órgão demonstram a potencialidade desta rede social para focar em educação em saúde, contribuindo para o empoderamento dos usuários, adaptando-se às ferramentas da contemporaneidade.

Neste aspecto, é importante atentar para os princípios trazidos pela Carta de Ottawa, documento que validou a importância de quaisquer estratégias que tenham como finalidade a capacitação dos sujeitos para o exercício da promoção da saúde, seja de maneira formal ou informal, via redes sociais on-line.

\section{REFERÊNCIAS}

ABREU, M. F.; MELO, E. M.; SILVEIRA, A. M. Contribuições do Planejamento Participativo Regionalizado (PPR) para a ampliação da cidadania e promoção da saúde. Saúde em Debate, Rio de Janeiro, v. 42, n. spe4, p. 107-119, 2018. Diaponível em: https://www.scielo.br/scielo.php?script=sci_arttext\&pid=S0103- 
11042018000800107. Acesso em: 10 set. 2019. doi: https://doi.org/10.1590/0103$11042018 s 408$

AGUIAR, A. Instagram: saiba tudo sobre esta rede social! Blog RockContent, São Paulo, ago/2018. Disponível em: https://rockcontent.com/blog/instagram/. Acesso em: 24 set. 2019.

APROBATO, V. C. Corpo digital e bem estar na rede Instagram: um estudo sobre as subjetividades e afetos na atualidade. Boletim - Academia Paulista de Psicologia, São Paulo, v. 38, n. 95, p. 157-164, 2018. Disponível em http://pepsic.bvsalud.org/scielo.php?script=sci_arttext\&pid=S1415-

711X2018000200003\&lng=pt\&nrm=iso. Acesso em: 10 ago. 2019.

BARROS, B. M. C. As novas mídias como instrumentos de resistência ao Controle da informação no Brasil: um olhar para os meios de comunicação e a luta pela democratização. CONFLUÊNCIAS - Revista Interdisciplinar de Sociologia e Direito, v. 20, n. 1, p. 5-22, 2018. Disponível em: https://periodicos.uff.br/confluencias/article/view/34548. Acesso em 04 abr. 2021. doi: https://doi.org/10.22409/conflu20i1.p526

BILOTTI, C. C.; NEPOMUCENO, L. D.; ALTIZANI, G. M.; MACUCH, R. S.; LUCENA, T. F. R. et al. m-Health no controle do câncer de colo do útero: prérequisitos para o desenvolvimento de um aplicativo para smartphones. Revista Eletrônica de Comunicação, Informação e Inovação em Saúde, v. 11, n. 2, p. 118, $2017 . \quad$ Disponível em: https://www.reciis.icict.fiocruz.br/index.php/reciis/article/view/1217. Acesso em: 10 ago. 2019. doi: https://doi.org/10.29397/reciis.v11i2.1217.

BRASIL. Ministério da Saude. Portaria № 2.446, de 11 de novembro de 2014. Redefine a Política Nacional de Promoção da Saúde (PNPS). Disponível em: http://bvsms.saude.gov.br/bvs/saudelegis/gm/2014/prt2446_11_11_2014.html.

Acesso em: 24 set. 2019.

CARVALHO, F. F. B.; COHEN, S. C.; AKERMAN, M. Reflecting on the established in Health Promotion to problematize 'dogmas'. Saúde em Debate [online], Rio de Janeiro, v. 41, n. spe3, p. 265-276, 2017. Disponível em: https://www.scielo.br/scielo.php?script=sci_abstract\&pid=S0103-

11042017000700265\&Ing=en\&nrm=iso\&tIng=en. Acesso em: 24 set. 2019. doi: https://doi.org/10.1590/0103-11042017s320.

CARVALHO, S. R.; GASTALDO, D. Promoção à saúde e empoderamento: uma reflexão a partir das perspectivas crítico-social pós-estruturalista. Ciência \& Saúde Coletiva, Rio de Janeiro, v. 13, n. suppl 2, p. 2029-2040, 2008. Disponível em: https://www.scielo.br/scielo.php?script=sci_arttext\&pid=S1413-81232008000900007. Acesso em: 24 set. 2019. doi: http://dx.doi.org/10.1590/S1413-81232008000900007

CASTELLS, M. O poder da identidade. São Paulo: Paz e Terra, 2000. 
CHIAPPERINO, L.; TENGLAND, P. A. Empowerment in healthcare policy making: Three domains of substantive controversy. Health Promotion Journal of Australia, v. 26, n. 3, p. 210-215, 2015. Disponível em: https://pubmed.ncbi.nlm.nih.gov/26650538/. Acesso em: 24 set. 2019. doi: 10.1071/HE15035

HOLMBERG, C.; BERG, C.; DAHLGREN, J.; LISSNER, L.; CHAPLIN, J.E. Health literacy in a complex digital media landscape: Pediatric obesity patients' experiences with online weight, food, and health information. Health Informatics Journal, v. 25, n. 4 , p. 1343-1357, mar/2018. Disponível em: https://pubmed.ncbi.nlm.nih.gov/29499615/. Acesso em: 30 set. 2018. doi: $10.1177 / 1460458218759699$

ISCHKANIAN, P. C.; PELICIONI, M. C. F. Comunicação em Saúde e Promoção de Práticas Integrativas e Complementares. In: In: PELICIONI, M. C. F.; MIALHE, F. L. Educação e Promoção da Saúde: teoria e prática. Rio de Janeiro: Santos, 2019. p. 95-104.

LA PEÑA, J. C. S. M. Capital social e saúde: análise crítica e implicações para a promoção da saúde na américa latina. In: PELICIONI, M. C. F.; MIALHE, F. L. Educação e Promoção da Saúde: teoria e prática. Rio de Janeiro: Santos, 2019. p. 41-59.

LIMA, S. G. P.; GUIMARÃES, K. M. F.; GERHARDT, P. C.; CASSIANO, A. C. M.; VERMELHO, S. C.. S. D. et al. A utilização de Redes Sociais Digitais na área da saúde. Saúde e Pesquisa, Maringá, v. 8, n. Edição Especial, p. 79-91, 2015. Disponível

https://periodicos.unicesumar.edu.br/index.php/saudpesq/article/view/3775. Acesso em: 30 set. 2019.

LIRA, A. G.; GANEN, A. P.; LODI, A. S.; ALVARENGA, M. S. Uso de redes sociais, influência da mídia e insatisfação com a imagem corporal de adolescentes brasileiras. Jornal Brasileiro Psiquiatria, Rio de Janeiro, v. 66, n. 3, p. 164-171, set/2017. Disponível

em:

http://www.scielo.br/scielo.php?script=sci_arttext\&pid=S0047-

20852017000300164\&Ing=en\&nrm=is. Acesso em:

SERRADILHA, A. F. Z.; DUARTE, M. T. C.; TONETE, V. L. P. Promoção da saúde por técnicos em enfermagem, na perspectiva de enfermeiros. Revista Brasileira de Enfermagem, Brasília, v. 72, n. 4, p. 1034-1042, 2019. Disponível em: https://www.scielo.br/scielo.php?pid=S0034-

71672019000400979\&script=sci_arttext\&tIng=pt. Acesso em: 15 ago. 2019. doi: https://doi.org/10.1590/0034-7167-2018-0552

MALTA, D. C.; REIS, A. A. C.; JAIME, P. C.; MORAIS NETO, O. L.; SILVA, M. M. A. et al. Brazil's Unified Health System and the National Health Promotion Policy: Prospects, results, progress and challenges in times of crisis. Ciencia e Saude 
Coletiva, v. 23, n. 6, p. 1799-1809, 2018. Disponível em: https://www.scielo.br/pdf/csc/v23n6/en_1413-8123-csc-23-06-1799.pdf. Acesso em: 10 ago. 2019. doi: DOI: $10.1590 / 1413-\overline{8} 1232018236.04782018$

MARCELINO, P. L.; SOUSA, J. P.; BRUCK, M. S. O uso do Facebook pelas operadoras de saúde brasileiras para estratégias de promoção da saúde: uma reflexão filosófica acerca da relação homem e mídia. Sapere Aude - Revista de Filosofia, v. 7, n. 13, p. 404-420, 2016. Disponível em: http://periodicos.pucminas.br/index.php/SapereAude/article/view/11931. Acesso em: 25 set. 2019. doi: https://doi.org/10.5752/P.2177-6342.2016v7n13p404.

MOREIRA, F. M.; PINHEIRO, M. M. K. Ministério da Saúde no Facebook: um estudo de caso da política de informação. Informação \& Informação, v. 20, n. 3, p. 147174 , 2015.

Disponível

em: http://www.uel.br/revistas/uel/index.php/informacao/article/view/19752.

Acesso em:25 out. 2019. doi: http://dx.doi.org/10.5433/1981-8920.2015v20n3p147.

PARACKAL, M.; PARACKAL, S.; EUSEBIUS, S.; MATHER, O. The use of Facebook advertising for communicating public health messages: a campaign against drinking during pregnancy in New Zealand corresponding author: JMIR Public Health Survell, v. 3, n. 3, e-49, 2017. Disponível em: https://www.ncbi.nlm.nih.gov/pmc/articles/PMC5571231/. Acesso em: doi: 10.2196/publichealth.7032

QUEMELO, P. R. V.; MILANI, D.; BENTO, V. F.; VIEIRA, E. R.; ZAIA, J. E. Literacia em saúde: Tradução e validação de instrumento para pesquisa em promoção da saúde no Brasil. Ciencia e Saude Coletiva, v. 33, n. 2, e00179715, 2017. Disponível em: https://www.scielo.br/scielo.php?pid=S0102311X2017000204001\&script=sci_abstract\&tlng=pt. Acesso em: 15 ago. 2019. doi: https://doi.org/10.1590/0102-311x00179715

TENGLAND, P. A. Empowerment: a goal or a means for health promotion? Medicine, Health Care and Philosophy, v. 10, n. 2, p. 197-207, 2007. Disponível em:

https://www.researchgate.net/publication/6708395_Empowerment_A_goal_or_a_me ans_for_health_promotion. Acesso em: 02 nov. 2019. doi: 10.1007/s $\overline{1} 10 \overline{19-006-}$ 9027-1

YAMAGUCHI, M. U.; BERNUCI, M. P.; PAVANELLI, G. C. Produção científica sobre a Política Nacional de Promoção da Saúde. Ciência e Saude Coletiva, v. 21, n. 6, p. 1727-1736, 2016. Disponível em: https://www.scielo.br/scielo.php?script=sci_arttext\&pid=S1413-

$81232016000601727 \& \operatorname{lng}=$ pt\&nrm=iso\&tlng=pt. Acesso em: 02 dez. 2019. doi: https://doi.org/10.1590/1413-81232015216.07462016. 